\title{
Investigating the dynamics of fission with low-fissility highly-excited nuclei
}

\author{
J. Benlliure ${ }^{1, a}$ Y. Ayyad ${ }^{1}$, A. Boudard ${ }^{2}$, E. Casarejos ${ }^{3}$, T. Enqvist ${ }^{4}$, F. Farget ${ }^{5}$, T. Kurtukian ${ }^{1}$, S. Leray $^{2}$, \\ M. Fernández ${ }^{1}$, P. Nadtochy ${ }^{6}$, D. Pérez ${ }^{1}$, R. Pleskac ${ }^{6}$, M.V. Ricciardi ${ }^{6}$, K.-H. Schmidt ${ }^{6}$, and C. Schmitt ${ }^{6}$ \\ ${ }^{1}$ Universidad de Santiago de Compostela, 15782 Santiago de Compostela, Spain \\ ${ }^{2}$ CEA-Saclay/IRFU, 91191 Gif-sur-Ivette, France \\ ${ }^{3}$ Universidad de Vigo, 36200 Vigo, Spain \\ ${ }^{4}$ Oulu Southern Institute and Department of Physics, University of Oulu, Finland \\ ${ }^{5}$ Grand Accélérateur National d'Ions Lourds, 14076 Caen, France \\ ${ }^{6}$ GSI Helmholtzzentrum für Schwereionenforschung GmbH, 64291, Germany
}

\begin{abstract}
Total fission cross sections and charge distributions of the final fission fragments have been measured at GSI in inverse kinematics for the reactions ${ }^{208} \mathrm{~Pb}+\mathrm{p}, \mathrm{d}$ at $500 \mathrm{~A} \mathrm{MeV}$ and ${ }^{182} \mathrm{Ta}+\mathrm{p}$ at $300,500,700$ and $1000 \mathrm{~A} \mathrm{MeV}$. These data are used to investigate dissipative and transient effects at low deformation and its possible dependences with temperature and fissility.
\end{abstract}

\section{Introduction}

The description of fission as a dissipative process, where the coupling between intrinsic (thermal) and collective (deformation) degrees of freedom is ruled by nuclear friction, was already proposed by Kramers soon after the discovery of this process [1]. This picture of fission was then completed by Grangé and collaborators [2] introducing the diffusion aspect by means of time-dependent transport equations leading to ground-to-saddle transient effects.

Dissipative effects were clearly established from pre- and post-scission particle emission [3], while pre-saddle transient effects have been deduced from fission cross sections [4-6]. However, the possible dependence of nuclear friction with temperature, deformation or fissility is still under debate [7, 8]. On another hand, the manifestation of transient effects may depend on the particular conditions of the fissioning nuclei $[9,10]$.

In this work we proposed to investigate fission induced in spallation reactions between protons and nuclei with relatively low fissility such as ${ }^{208} \mathrm{~Pb}$ and ${ }^{181} \mathrm{Ta}$. These reactions produce highly excited nuclei with low angular momentum and small modifications of the ground deformation of the fissioning systems. Under these conditions one expects clear manifestations of transient effects.

\footnotetext{
ae-mail: j.benlliure@usc.es
}

This is an Open Access article distributed under the terms of the Creative Commons Attribution License 2.0, which permits unrestricted use, distribution, and reproduction in any medium, provided the original work is properly cited. 


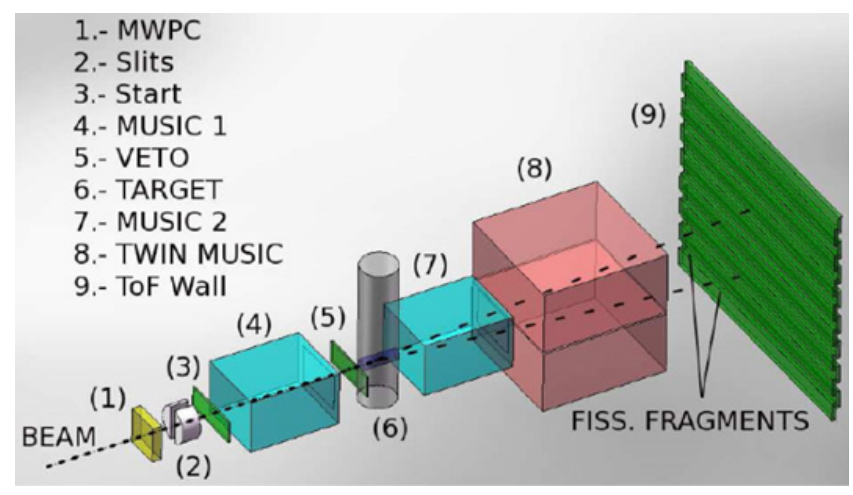

Figure 1. Schematic representation of the experimental setup.

Moreover, the present data together with the fission of actinides previously investigated [4, 6], will allow to cover a large range in fissility and initial deformation.

The main observables we propose for this investigation are total fission cross sections and the charge distributions of the fission fragments. In order to optimize these measurements we investigate the reactions in inverse kinematics, enabling a better identification of the fission fragments, and therefore, a more accurate determination of the fission cross sections and charge characterization of the fission fragments.

\section{The experiment}

The experiment was performed at the GSI facilities in Darmstadt (Germany). The SIS18 synchrotron delivered beams of ${ }^{208} \mathrm{~Pb}$ accelerated at $500 \mathrm{~A} \mathrm{MeV}$ and ${ }^{181} \mathrm{Ta}$ at $300,500,700$ and $1000 \mathrm{~A} \mathrm{MeV}$. The typical intensity of those beams was around $10^{5}$ ions/pulse with a pulse duration of $5 \mathrm{~s}$. Beams impinged into a cryogenic target filled with hydrogen $\left(85 \mathrm{mg} / \mathrm{cm}^{2}\right)$ and deuterium $\left(201 \mathrm{mg} / \mathrm{cm}^{2}\right)$ encapsulated with $30 \mu \mathrm{m}$ titanium windows. The two fission fragments, emitted forward because of the kinematics of the reaction were detected and identified in atomic number using a dedicated detection setup sketched in Figure 1.

As indicated in Figure 1, the cryogenic target was surrounded by two multi-sampling ionisation chambers (MUSIC) used to identify the reactions taking place in the target from those produced in the other layers of matter placed along the beam axis, up- and down-stream the target position. A multi-wire proportional chamber (MWPC) and a scintillator with a central hole (veto) were used to align the beam at the target position. In measurements with the ${ }^{208} \mathrm{~Pb}$ beam, the two fission fragments were identified in atomic number from their energy lost in a twin multi-sampling ionisation chamber (twin MUSIC) and their velocity determined from the time of flight between a plastic scintillator placed up-stream the target (star) and a scintillator wall (ToF wall) located at $5 \mathrm{~m}$ from the target.

The detection efficiency of this setup was evaluated to be around $90 \%$, being the main limitation the common horizontal cathode of the twin multi-sampling ionisation chamber. A detailed description of the experimental setup and the data analysis can be found in Ref. [11].

\section{Results}

\subsection{Charge distributions of the fission fragments}

In the measurements with the ${ }^{208} \mathrm{~Pb}$ beam we could determine the atomic number of both fission fragments from the signals registered by the twin-MUSIC [11]. The sum of the atomic numbers of 


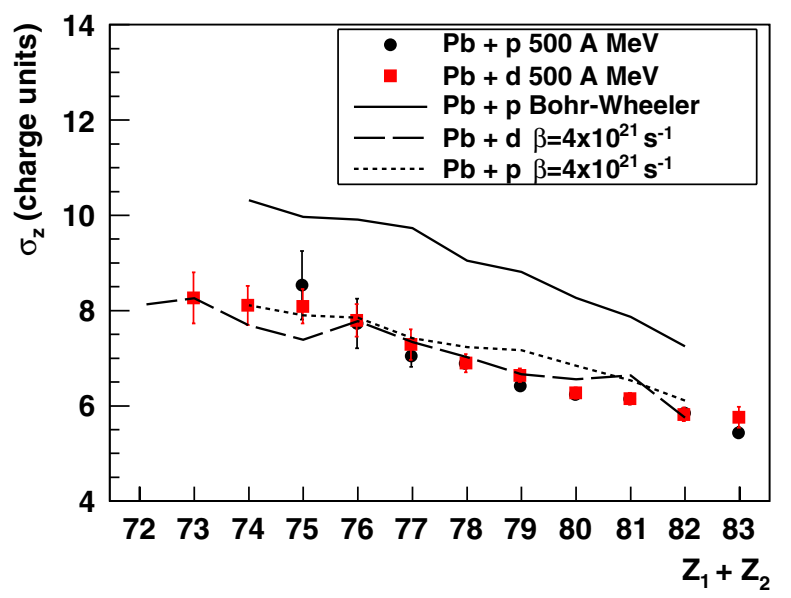

Figure 2. Width of the charge distribution of the fission fragments obtained with hydrogen (points) and deuterium (squares) as a function of the atomic numbers of the two fragments.

both fragments has been proposed as a good indicator of the excitation energy of the fissioning system at ground deformation [4]. Moreover, the width of the charge distribution of the fission fragments has been shown to depend on the temperature of the fissioning system at saddle [4, 7].

On another hand, spallation reactions provides optimal conditions for the investigation of groundto-saddle dissipative effects. Indeed, reactions induced by relativistic protons on lead are expected to produce highly excited prefragments with low angular momentum, while keeping an almost spherical ground configuration [9]. Therefore we have used the two observables measured in this experiment, the sum of the atomic numbers of the two fission fragments and the width of the charge distribution, to investigate ground-to-saddle transient effects.

In Figure 2 we represent the partial width of the charge distributions of the fission fragments as a function of the sum of the atomic number of both fragments for the reactions induced on hydrogen (points) and deuterium (squares). As can be seen, the partial width of the charge distributions obtained with both targets for the same value of the sum of the atomic numbers of both fragments is similar. This result indicates that this observable does not depend of the entrance channel of the reaction but on the properties of the fissioning system, in this case on the temperature at saddle.

The investigation of dissipative effects requires model calculations describing the fission width. In Figure 2 we show results obtained with two models, a purely statistical model based on the BohrWheeler prescription [12], and calculations obtained with the ABLA code [13], where an analytical solution of the time-dependent Fokker-Plank equation describing the coupling between collective (deformation) and intrinsic (excitation energy) degrees of freedom in terms of a reduced friction coefficient $\beta[14,15]$. Both calculations are based on the fission barriers calculated according to Sierk [16] and deformation-dependent level densities [17] that together with the low angular momentum induced by these reactions fulfils the criteria proposed in [10].

The predictions obtained with both models are also shown by lines in Figure 2. The results obtained with the statistical model (solid line) clearly overestimate the width of the charge distributions reflecting larger values of the excitation energy of the fissioning system at saddle. Contrary, calculations based on dynamical description of the fission process with a value of the reduced dissipation parameter $\beta$ around $4 \cdot 10^{21} \mathrm{~s}^{-1}$ provide a good description of the measured widths of the charge distributions in reactions induced with protons (dashed line) and deuterons (dashed-dotted line). These results are in good agreement with previous investigations based in the use of fragmentation $[4,7]$ and spallation reactions $[5,6]$. 


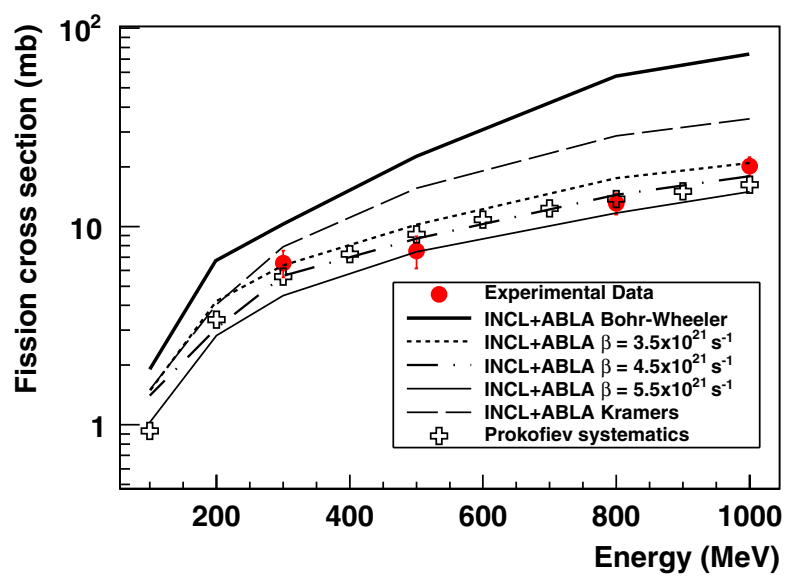

Figure 3. Total fission cross sections for the reaction ${ }^{181} \mathrm{Ta}+\mathrm{p}$ at different kinetic energies.

\subsection{Total fission cross sections}

The total fission cross section could be obtained with the ${ }^{208} \mathrm{~Pb}$ beam impinging hydrogen and deuterium at $500 \mathrm{~A} \mathrm{MeV}$ but also with the ${ }^{181} \mathrm{Ta}$ beam impinging hydrogen at 300, 500, 700 and $1000 \mathrm{~A} \mathrm{MeV}$. The absolute cross sections were accurately determined by normalizing the recorded number of fission events to the number of incoming lead projectiles and the number of target nuclei. The number of fissions was obtained from the energy loss signals registered at both sides of the twin multi-sampling ionisation chamber. In such a plot, a clear region of events with similar values of the energy lost in both chambers allowed us to clearly identify and separate fission events from other competing de-excitation channels. The number of beam projectiles could be obtained from the MUSIC chamber placed in front of the target where the energy lost by the incoming nuclei made it possible to identify lead projectiles from other nuclear species produced in reactions of the lead projectiles with the layers of matter placed up-stream the target. The number of target atoms was determined from a precise measurement of the target thickness.

The excitation function obtained with the ${ }^{181}$ Ta beam was used to investigate the onset of transient effects [18]. In Figure 3 we report the measured total fission cross sections as a function of the beam energy. In this figure we also represent the results obtained with different model calculations, in particular with a purely statistical model (solid line), a time-independent dynamical model (long dashed line) and three time-dependent dynamical calculations with different values of the nuclear viscosity $\beta=3.510^{21} \mathrm{~s}^{-1}$ (dashed line), $\beta=4.510^{21} \mathrm{~s}^{-1}$ (dashed-dotted line) and $\beta=5.510^{21} \mathrm{~s}^{-1}$ (thin solid line). As can be seen, purely statistical calculations overestimate the measured total fission cross sections while a time-dependent dynamical calculation with a value of the nuclear viscosity parameter around $4.510^{21} \mathrm{~s}^{-1}$ seems to better describe de data. Moreover, time-independent dynamical calculation with the same values of the nuclear viscosity also reproduce rather well data at kinetic energies below $300 \mathrm{MeV}$. We conclude, then, from this analysis that transient effects only manifest at excitation energies above $150 \mathrm{MeV}$.

In Figure 4 we complete the total fission cross sections measured in this work with others obtained in proton induced fission at energies above $600 \mathrm{MeV}$ on different target nuclei as a function of the fissility [19-24]. We also compare these total fission cross sections with time-dependent dynamical calculations with different values of the nuclear viscosity parameter. From this comparison we can conclude that the nuclear viscosity parameter does not seem to depend very much on the fissility of the nuclei, neither on their initial deformation since we can reproduce all cross sections, covering a large range in fissility and 


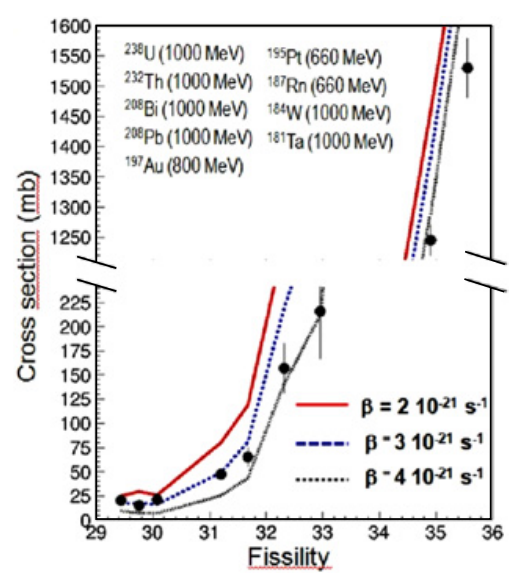

Figure 4. Total fission cross sections of proton induced fission at energies above $600 \mathrm{MeV}$ on different target nuclei as a function of the fissility.

initial deformation, with a value of the nuclear viscosity between $310^{21} \mathrm{~s}^{-1}$ and $510^{21} \mathrm{~s}^{-1}$. Moreover, this small variation could still be subject to entrance channel effects or the competition of fission with the emission of intermediate-mass fragments that should be further investigated.

\section{Conclusions}

A new experiment based on the inverse kinematic technique and an advanced detections setup with high detection efficiency allowed us to determine the total fission cross sections in reactions induced with ${ }^{181} \mathrm{Ta}$ and ${ }^{208} \mathrm{~Pb}$ beams at different energies impinging a cryogenic target filled with hydrogen and deuterium. In the case of the ${ }^{208} \mathrm{~Pb}$ experiment we could also determine the atomic number of both fission fragments with high accuracy. These data allowed to investigate the dynamics of fission at high excitation energy because of the optimal conditions of the fissioning systems, low angular momentum and deformation.

The sum of the atomic numbers of both fissioning systems and the width of their charge distribution was used as indicators of the temperature of the fissioning systems at ground deformation and at saddle. On the other hand, total fission cross sections were used to investigate dependences of the nuclear viscosity with deformation and fissility.

This work was partially supported by the European Commission under project FI6W-CT-2004-516520 EUROTRANS, by the Spanish Ministry of Research and Innovation under the grant FPA2007-62652 and BES2008-005553, the project consolider-CPAN CSD2007-00042 and the Regional Government of Galicia under the program "grupos de referencia competitiva" 2006/46.

\section{References}

[1] H.A. Kramers, Physica 7, 284 (1940)

[2] P. Grangé, Li Jun-Qing and H.A. Weidenmüller, Phys. Rev. C 27, 2063 (1983)

[3] D. Hilscher and H. Rossner, Ann. Phys. 17, 471 (1992) 
[4] B. Jurado et al., Phys. Rev. Lett. 93, 072501 (2004)

[5] J. Benlliure et al., Nucl. Phys. A 700, 469 (2002)

[6] J. Benlliure et al., Phys. Rev. C 74, 014609 (2007)

[7] C. Schmitt et al., Phys. Rev. C 81, 064602 (2010)

[8] D. Jacquet, M. Morjean, Prog. Part. Nucl. Phys. 63, 155 (2009)

[9] B. Jurado et al., Nucl. Phys. A 757, 329 (2005)

[10] J.P. Lestone, S.G. McCalla, Phys. Rev. C 79, 044611 (2009)

[11] K.-H. Schmidt et al., Phys. Rev. C 87, 034601 (2013)

[12] N. Bohr, J.A. Wheeler, Phys. Rev. 56, 426 (1939)

[13] A. Kelic, M.V. Ricciardi and K.-H. Schmidt, ArXiv:0906.4193 (2009)

[14] B. Jurado et al., Phys. Lett. B 553, 186 (2003)

[15] B. Jurado et al., Nucl. Phys. A 747, 14 (2005)

[16] A.J. Sierk, Phys. Rev. C 33, 2039 (1986)

[17] A.V. Ignatyuk et al. Yad. Fiz. 21, 1185 (1975) [Sov. J. Nucl. Phys. 21, 612 (1975)]

[18] Y. Ayyad et al. in preparation

[19] A.A. Kotov et al., Sov. J. Nucl. Phys. 19, 756 (1974)

[20] V.A. Konshin et al., Sov. J. Nucl. Phys. 2, 489 (1969)

[21] J. Benlliure et al., Nucl. Phys. A 683, 513 (2001)

[22] T. Envist et al., Nucl. Phys. A 686, 481 (2001)

[23] V.I. Yurevich et al., Phys. At. Nucl. 65, 1383 (2002)

[24] A.A. Kotov et al., Phys. Rev. C 74, 034605 (2006) 